\title{
LAS DIFERENCIAS DE GÉNERO EN LA NEGOCIACIÓN DE LOS ACUERDOS DE COOPERACIÓN Y SU INFLUENCIA SOBRE LOS RESULTADOS DEL PROCESO
}

Carmen Saorín-Iborra ${ }^{1}$

Terese Canet-Ginev²

\begin{abstract}
Resumen: Las relaciones entre género y comportamiento, así como el cumplimiento del estereotipo de comportamiento esperado es una cuestión clave sobre la que el trabajo que presentamos intenta profundizar. En concreto, analizamos la influencia de las diferencias de género y del comportamiento derivado de estas diferencias sobre los resultados de los procesos de negociación. Con el objeto de controlar la influencia del contexto en el que el proceso de negociación se produce, nos centramos en el análisis de las negociaciones de acuerdos de cooperación. Este tipo de acuerdos se caracterizan por un elevado grado de ambigüedad estructural, las negociaciones son de suma variable y los agentes que actúan en ellas lo hacen en nombre del colectivo al que representan. En esta situación es cuando las diferencias de género se hacen patentes, lo cual nos conducirá a reflexionar sobre la composición idónea del equipo negociador, la cual debería orientarse, en nuestra opinión, hacia un perfil de género "andrógino" capaz de adoptar comportamientos tanto cooperativos como competitivos en función de la situación. Estas reflexiones quedan recogidas en las tres proposiciones formuladas y en la posterior discusión final.
\end{abstract}

Palabras clave: género, comportamiento negociador, resultados de la negociación, equipo negociador, alianzas estratégicas.

\section{INTRODUCCIÓN}

El análisis de las diferencias de género y su influencia en el campo de la Dirección Estratégica ha experimentado un gran auge en los últimos años, siendo prueba de ello la proliferación de trabajos al respecto en las dos últimas décadas. El fenómeno de la influencia de género ha sido abordado desde diferentes perspectivas teóricas, aunque principalmente desde la psicología social y desde el feminismo. Pero ha sido en los últimos años de la década de los noventa cuando ha surgido un creciente interés sobre los temas de género, proveniente de otras áreas de las ciencias sociales como la Dirección

\footnotetext{
${ }^{1}$ Universidad de Valencia- España. E.Mail: Carmen.Saorin@uv.es

${ }^{2}$ Universidad de Valencia- España. E.Mail: Teresa.Canet@uv.es
} 
Estratégica y el Comportamiento Organizativo. En este sentido, han ido apareciendo trabajos que estudian este fenómeno analizando, entre otras cuestiones, las relaciones entre el género y la existencia de un espíritu empresarial emprendedor (ALMER, 2000; ROSENTHAL y RODRIGUES, 2000), el efecto de las diferencias de género sobre los procesos de toma de decisiones estratégicas (VERHEUL, RISSEEUW y BARTELSE, 2002; KLENKE, 2003) o la influencia de género en los procesos de negociación y resolución de conflictos (WALTER, STUHLMACHER y MEYER, 1998; BREWER, MITCHELL y WEBER, 2002).

La influencia del comportamiento en las diferentes relaciones y procesos organizativos es una cuestión clave en el marco de esa línea de investigación. Es más, las relaciones entre género y comportamiento, así como el cumplimiento del estereotipo de comportamiento esperado es una cuestión clave sobre la que la literatura más reciente intenta desarrollar nuevos planteamientos.

En este trabajo nos centramos, en particular, en esta última corriente de investigación. En este sentido, estudiamos el comportamiento negociador en un contexto determinado como son los acuerdos de cooperación. Los procesos de negociación de los acuerdos de cooperación suponen un área de investigación casi olvidada. Por tanto, tratando de compensar la ausencia de trabajos que se observa en la literatura de estrategia sobre este tema $y$, al mismo tiempo, intentando responder a la creciente preocupación por la influencia del género en el mundo empresarial, el objetivo de nuestra propuesta es analizar cómo las diferencias de género pueden afectar a los procesos de negociación de los acuerdos de cooperación (comportamiento mostrado y resultados obtenidos) y, como consecuencia, estudiar las implicaciones que puede tener dicha influencia en la elección del equipo negociador.

Con tal finalidad, el trabajo se ha estructurado en cuatro grandes partes. En la primera delimitamos el contexto objeto de estudio indicando qué entendemos por acuerdo de cooperación, sus características comunes y las distintas modalidades que existen. A continuación, en la segunda parte, se argumenta la importancia de los procesos negociadores de los acuerdos de cooperación por sus implicaciones en la posterior implantación de la estrategia. En este sentido, se realiza una breve revisión de la literatura sobre negociación, intentando delimitar qué se entiende por comportamiento negociador, los tipos de comportamientos que se distinguen y el efecto de éstos sobre la consecución de un resultado u otro en las negociaciones. En una tercera parte presentamos el estado de la cuestión en relación al análisis del impacto del género en los procesos de negociación (comportamiento negociador mostrado y los resultados obtenidos). Finalmente, en la última parte se analiza el impacto de las diferencias en género en los procesos negociadores en un contexto específico, nuestro objeto de estudio, los acuerdos de cooperación. En este sentido, se proponen una serie de relaciones que constituirán hipótesis a contrastar en futuras investigaciones.

\section{LOS ACUERDOS DE COOPERACIÓN: CONTEXTO DE ANÁLISIS}

Desde los años 80 se observa una tendencia creciente en el desarrollo de acuerdos de cooperación entre empresas, como una de las mejores opciones estratégicas para enfrentarse a las necesidades de flexibilidad exigidas por el entorno competitivo actual.

De una manera sencilla, un acuerdo de cooperación se define como un acuerdo por 
el que dos o más organizaciones comparten recursos y actividades con un objetivo estratégico común (JONSON et al, 2005). De la definición anterior se extraen una serie de características comunes a todo acuerdo de cooperación. Así, el contenido del acuerdo debe ser explícito, ya que es fundamental la implicación real y desde las fases previas de las empresas involucradas con la finalidad de resultar una relación con éxito. Sin embargo, aunque la mayor parte de los acuerdos de cooperación se basan en acuerdos escritos y contratos formales, acuerdos únicamente verbales pueden también tener lugar, siendo éstos últimos perfectamente válidos (MENGUZZATO, 1992).

Por otro lado, la duración de los acuerdos de cooperación es limitada en el tiempo, y lo razonable es que se desarrollen en el medio o largo plazo. Este periodo es superior al de una transacción de mercado e inferior a la vida de la empresa. Tras alcanzar los objetivos previamente fijados en el acuerdo, éste se dará por finalizado.

Como tercera característica, cabe destacar que este tipo de acuerdos afectan únicamente a una parte de la actividad global de los socios. De este modo, el acuerdo puede establecerse en torno a una de las funciones básicas de la empresa (investigación, desarrollo de procesos y/ productos, distribución producción, marketing...), bien para la realización conjunta de un proyecto específico, o bien para un mejor desarrollo de una o varias actividades concretas de la empresa.

En el contexto del acuerdo de cooperación, ambos socios intentan conseguir un objetivo común. Por este motivo resulta fundamental conseguir un buen nivel de coordinación entre las decisiones que los socios adoptan y las acciones que desarrollan para ponerlas en práctica; al menos se requiere la estrecha colaboración de aquellos directamente relacionados con la actividad objeto del acuerdo de cooperación. Así pues, será necesario especificar con detalle las aportaciones que cada empresa debe hacer, así como la asignación de tareas a los diferentes participantes en el acuerdo, de manera que sea posible garantizar la reciprocidad entre los socios.

No obstante, a pesar de las mencionadas características comunes a todo acuerdo de cooperación, bajo este término encontramos diferentes modalidades o tipos de relaciones las cuales poseen, a su vez, características particulares. En este sentido, la literatura sobre estrategia (JONSON et al, 2005) distingue entre los siguientes tipos 0 modalidades de acuerdos de cooperación, ordenados de mayor a menor vinculación de las partes en el acuerdo:

Joint Venture: Consiste en un acuerdo mediante el cual dos o más empresas independientes deciden crear una nueva, distinta a las anteriores, pero cuyo capital social es compartido por los socios; reciben en compensación los resultados (beneficios, know-how...) generados por la actividad de dicha empresa. Se utiliza con frecuencia como vía de entrada en países como China.

Consorcios: Son acuerdos cuyo fin es llevar a cabo un trabajo conjunto. Suelen centrarse en un proyecto en particular. Con relación a este tipo de acuerdos, Menguzzato (1992) señala que tienen lugar cuando las características del trabajo hacen que una empresa por sí sola no tenga capacidad técnica, comercial y/o financiera para realizarlo. Normalmente se realizan entre competidores y se suelen dotar de órganos comunes que tienen como misión esencial la coordinación de actividades de los partenaires, y en ciertos casos, también la de representación frente a terceros.

Franquicia: En esta modalidad una empresa -el franquiciador- a cambio de determinadas compensaciones económicas, cede a otra(s) empresa(s) -franquiciado- el derecho a comercializar y/o fabricar un producto ya acreditado. El franquiciador suele ser responsable de la imagen de marca, de la política de marketing y, en algunos casos de la de formación. 
Licencia: es común en industrias relacionadas con la investigación científica y la innovación, y permite el derecho a fabricar un producto patentado a cambio del pago de una tasa o cuantía económica.

Subcontratación: En esta forma de cooperación, la empresa contratista, encarga a otra empresa -la subcontratada- la realización de determinados servicios, o de parte de un proceso. Suelen ser acuerdos contractuales, al igual que los dos anteriores.

Redes: Las redes interempresariales se encuentran en el otro extremo con respecto al criterio de vinculación, y se trata de dos o más organizaciones que colaboran sin que sea necesario establecer relaciones formales, ya que todas obtendrán ventajas resultado de la colaboración.

Menguzzato (1992) añade otras formas de acuerdo de cooperación, como los acuerdos de coproducción, que tienen por objeto la producción en común de un producto por dos o más empresas; el spin-off, cuando una empresa impulsa y apoya la creación de otra por personas de su plantilla, que así se independizan, con el fin de que esta nueva empresa se convierta en un partenaire que realice la actividad que llevaban a cabo en la otra bajo la responsabilidad de dichas personas; los acuerdos de $I+D$, mediante los cuales, unas empresas independientes entre sí, se comprometen a poner en común una parte de sus recursos y habilidades para llevar a cabo actividades conjuntas de investigación y desarrollo; o los acuerdos de Marketing, como los de distribución, los de compra conjunta y los de marketing cruzado.

Según esta misma autora, todas las modalidades indicadas pueden, a su vez, agruparse en función de si existe o no participación en el capital distinguiéndose respectivamente entre acuerdos de cooperación estructurados y no estructurados.

\section{LOS PROCESOS DE NEGOCIACIÓN: COMPORTAMIENTO NEGOCIADOR Y RESULTADOS}

Los procesos de negociación de los acuerdos de cooperación son una cuestión clave, por su influencia en la correcta implantación de la estrategia y la performance resultante. Tal y como argumenta Valdés (1998), los procesos de negociación suponen la culminación de las fases anteriores de la alianza y es donde deben establecerse las bases satisfactorias para asegurar resultados positivos en el futuro. De forma similar Menguzzato (1992) expresa que las negociaciones constituyen un proceso en el que no sólo se define el contenido formal del acuerdo, sino en el que las partes aprenden a conocerse, creándose expectativas sobre el comportamiento futuro del socio y, por tanto, una relación de confianza entre los partners. En este sentido, se defiende que el resultado de la negociación influye en la posterior implantación de la estrategia. De este modo, el proceso negociador se configura como nexo entre la formulación de la estrategia y su implantación. Por otro lado, la literatura de negociación demuestra que el resultado de la negociación viene determinado principalmente por el comportamiento mostrado por las partes negociadoras (RUBIN y BROWN, 1975; PRUITT, 1981; LAX y SEBENIUS, 1986; FISHER, URY y PATON, 1983; WEISS, 1993).

El comportamiento negociador se define como el conjunto de tácticas de comunicación visibles o acciones (verbales y no verbales) que cada negociador dirige al oponente (RUBIN y BROWN, 1975; PUTNAM, 1990; ADLER ET AL., 1992; RAO y 
SCHMIDT, 1998, SAORÍN, 2004). En este sentido, las partes pueden adoptar distintos tipos de comportamientos en la negociación, en función de cuál es el tipo de tácticas que desean aplicar. La literatura distingue entre los comportamientos negociadores integrativos y competitivos.

Los comportamientos integrativos implican el intercambio de información de forma abierta, clara y honesta. De este modo, los negociadores que muestran este tipo de comportamientos utilizan tácticas como las preguntas y las auto-revelaciones (ADLER et al., 1992) ${ }^{3[1]}$ estableciéndose, consecuentemente, una comunicación efectiva entre las partes. Por el contrario, los comportamientos competitivos de negociación implican la ocultación de información, principalmente aquella de carácter básico. Cuando las partes muestran comportamientos competitivos, la interacción comunicativa es inefectiva porque éstas tienden a utilizar tácticas como demandas, negativas, amenazas, solapamientos en la conversación, contactos físicos, etc (ADLER et al., 1992).

Trabajos previos han demostrado la relación positiva existente entre los comportamientos negociadores integrativos y la consecución de acuerdos mutuamente satisfactorios (RUBIN y BROWN, 1975; PRUITT, 1981; ADLER et al., 1992; TJOSVOLD et al., 1999). Este tipo de comportamientos facilita la creación de confianza entre las partes, la reducción de incertidumbre (expectativas) y, consecuentemente, permiten la satisfacción de intereses mutuos así como el establecimiento y mantenimiento de un compromiso a largo plazo hacia la relación negociada (MUNDUATE y MEDINA, 2005b). Por esta razón se argumenta que los comportamientos negociadores integrativos son los deseables en los acuerdos de cooperación, debido a sus efectos positivos sobre la implantación y la posibilidad de mantener el compromiso a medio o largo plazo (RING y VAN DE VEN, 1994; ARIÑO y GARCÍA, 1996; ARIÑO y DE LA TORRE, 1998). Recordemos que por definición, los acuerdos de cooperación tienen como objetivo establecer y mantener la relación a medio o largo plazo.

La relación inversa se argumenta respecto a los comportamientos competitivos. Se demuestra que estos comportamientos se asocian al fracaso de la negociación porque dificultan la consecución de un acuerdo (RUBIN y BROWN, 1975; URY et al., 1988; WEISS, 1997; TJOSVOLD et al., 1999; MUNDUATE y MEDINA, 2005b). Sin embargo,

\footnotetext{
${ }^{3[1]}$ Estos autores, partiendo de la propuesta de Graham (1985: 88), caracterizan los distintos comportamientos negociadores en función, entre otras, de una serie de tácticas verbales como son:

Promesa: Afirmación en la que el negociador indica su intención de dar al partner una consecuencia de refuerzo que éste valorará como positiva o grata.

Amenaza: Similar a la promesa pero en la que la consecuencia de refuerzo se valorará como negativa o castigo.

Recomendación: Afirmación en la que el negociador predice que alguna consecuencia del entorno grata le ocurrirá al partner, no estando la misma bajo el control del primero.

Aviso: Similar a la recomendación pero siendo la consecuencia negativa.

Recompensa: Afirmación del negociador que se considera que conllevará consecuencias positivas para el partner.

Penalización: Similar a la recompensa pero en este caso las consecuencias son negativas.

Apelación positiva y normativa: Afirmación en la que el negociador indica que el comportamiento pasado, presente y futuro del partner estuvo, está o estará en conformidad con las normas sociales.

Apelación negativa y normativa: Similar al anterior pero en la que se manifiesta que el comportamiento no estuvo, está o estará en conformidad con las normas sociales.

Compromiso: Afirmación del negociador acerca de que sus ofertas no serán inferiores o superiores a un nivel determinado.

Auto-revelación: Afirmación en la que el negociador revela información sobre él mismo.

Pregunta: Afirmación en la que el negociador requiere al socio que revele información.

Demanda: Afirmación en la que el negociador sugiere que el socio tenga un determinado comportamiento.
} 
en caso de alcanzar un acuerdo, éste conllevaría la insatisfacción de al menos una de las partes, con el consiguiente efecto negativo sobre la implantación y performance de la estrategia. Este efecto se muestra con más fuerza cuando es crucial mantener entre las partes una colaboración y compromiso a largo plazo (MUNDUATE y MEDINA, 2005b). Pero en esta situación se dificulta el mantenimiento de un compromiso hacia la relación objeto de negociación.

Durante la interacción negociadora, las partes muestran distintos tipos de comportamientos con orientación integrativa o competitiva (LAX y SEBENIUS,1986). No obstante, siguiendo la argumentación de Roure (1997), siempre predominará un comportamiento sobre el resto, influyendo éste sobre la determinación del resultado del proceso alcanzado.

\section{INFLUENCIA DEL GÉNERO EN LOS PROCESOS DE NEGOCIACIÓN}

Desde la psicología social se ha tratado de explicar cómo el género influye tanto sobre los procesos de negociación como sobre el resultado alcanzado en estos procesos. Sin embargo, en los estudios previos encontramos una evidencia contradictoria al respecto. Lewicki y Litterer (1985) no encontraron ninguna característica de género que estuviera relacionada con el éxito de la negociación. Por otro lado, Thomas (2000) afirma que las mujeres demuestran un menor deseo de incrementar sus responsabilidades y autopromoción en la empresa; Graves y Elsass (2005) señalan que los efectos del sexo pueden influir y constituir potentes determinantes de las experiencias en equipos de los individuos (en nuestro caso, los equipos de negociación); y Koretz (2001) observa diferencias en el comportamiento mostrado por los hombres y las mujeres en la negociación (por ejemplo, las mujeres son más maleables y flexibles que los hombres).

Graves y Elsass (2005) afirman, de igual modo, que cuando las mujeres trabajan en equipos se espera que muestren comportamientos de subordinación, mientras que de los hombres se espera un comportamiento dominante.

Walter, Stuhlmacher y Meyer (1998) plantean, a través de su revisión teórica, que las diferencias de género tradicionales en las negociaciones - las mujeres adoptando una actitud más cooperativa y los hombres un rol más competitivo—no aparecen reflejadas de forma consistente en los diferentes estudios analizados en su trabajo, enfatizando, a su vez, que la metodología utilizada en el análisis podría haber afectado los resultados obtenidos en dichos estudios. Fernandez, Medina y Dorado (2005) realizan una revisión de la literatura, e indican que una explicación posible a las diferencias obtenidas en los resultados de los trabajos analizados podría estar en el hecho de que se ha omitido el estudio de los factores contextuales que afectan a los procesos de negociación (CARNEVALE y PRUITT, 1992). También podría influir el planteamiento de que son los roles de género y no sólo el sexo los que guiarían el comportamiento de los actores bajo circunstancias contextuales específicas (BREWER, MITCHELL y WEBER, 2002).

Por último, Parghi y Murphy (1999) llevan a cabo una revisión de la literatura centrándose en aquellos estudios que analizan la influencia del género en la resolución del conflicto y la negociación. En este estudio, los autores señalan que los trabajos existentes (HALPERN y PARKS, 1996; NEU, GRAHAM y GILLY, 1988; PRUITT, CARNEVALE, FORCEY y VAN SLYCJ, 1986; SMELTZER y WATSON, 1986) observan diferencias potenciales de género en los comportamientos negociadores pero que, por 
otro lado, no existe evidencia de los efectos del género en los resultados de los procesos de negociación, en particular, en el grado de satisfacción obtenido por los negociadores.

Partiendo de la premisa de Lewin (1936), por la cual el comportamiento social se considera como el resultado de la interacción entre el individuo y la situación, Riley y McGuinn (2002) proponen, en un trabajo reciente, el análisis de la relación entre las diferencias de género y la negociación desde un enfoque situacional o "contextual". Si bien, previamente Thompson (1990) ya había argumentado la necesidad de aplicar un enfoque contingente ante su validez de predicción en comparación con los modelos de efectos directos, la ausencia de una base teórica dificultó la identificación de cualquier interacción contextual y su consiguiente propuesta teórica.

Aunque el análisis realizado por Riley y McGuinn (2002) parte del concepto de la conocida "amenaza de estereotipo"4 [2] (STEELE y ARONSON, 1995; STEELE, 1997), éstos argumentan que la situación o contexto puede favorecer o eliminar la aparición de las diferencias individuales y, consecuentemente, las expectativas de comportamiento y de performance propias de esas diferencias. En este sentido, y partiendo de investigaciones previas centradas en el comportamiento social, proponen dos moderadores contextuales en relación al impacto de las diferencias de género sobre los procesos de negociación (en concreto sobre el comportamiento y los resultados esperados). Dichos factores moderadores son la fuerza psicológica de la situación (MISCHEL, 1977) y la presencia de factores desencadenantes o impulsores (SNYDER y ICKES, 1985), los cuales han sido adaptados por los autores al contexto de la negociación denominándolos, respectivamente, ambigüedad estructural y desencadenantes de la influencia de género.

En opinión de Mischel (1977), la fuerza psicológica de la situación puede medirse analizando el grado en el que la situación puede llevarnos a esperar un patrón de respuesta adecuada. La fortaleza de la situación depende, por un lado, del grado en que existen pautas o ejemplos de conducta que guían el comportamiento de los actores y, por otro lado, del grado de definición o estructuración de la situación. En este sentido, Snyder y Ickes (1985) distinguen entre situaciones fuertes y débiles. Las situaciones fuertes son aquellas que están muy bien definidas y estructuradas y guían el comportamiento de los agentes, mientras que las situaciones débiles son no estructuradas y ambiguas. En este sentido, existen varios estudios que señalan cómo estas situaciones ambiguas impulsan y conllevan la improvisación ante la inexistencia de un guión prescrito de comportamiento (Dykman, ABRAMSON, ALLOY y HARTLAGE, 1989; LAMBERT y WEDELL, 1991; MACLEOD Y COHEN, 1993; HOCK, KROHNE Y KAISER, 1996). Bajo la improvisación, las personas se comportan siguiendo su propio pensamiento, valores y esquemas cognitivos y es en esa situación cuando los estereotipos de género aparecen debido a que se producen juicios sesgados, tanto sobre los otros como sobre uno mismo (FISKE y TAYLOR, 1991; BABCOCK y LOEWENSTEIN, 1997).

En su aplicación al contexto de la negociación, Riley y McGuinn (2002) denominan a este primer factor contextual como "la ambigüedad estructural de la negociación" y la definen como el grado en el que puede variar la interpretación de una parte de la estructura económica de la negociación (RILEY Y MCGUINN, 2002: 15). Así, esta ambigüedad hace referencia a si la probabilidad respecto a las alternativas o a la naturaleza de las mismas es conocida o no. Entendemos que esta situación es la que habitualmente se

\footnotetext{
4[2] "La existencia de un estereotipo implica que algo o alguna característica de alguien que lo configure hace que el estereotipo sea más plausible como auto-caracterización a los ojos ajenos y quizás incluso a los propios" (STEELE y ARONSON, 1995; STEELE, 1997; citados en KRAY, GALINSKY y THOMPSON, 2002: 388).
} 
produce en las negociaciones "reales", al poder existir diferentes niveles de ambigüedad en función del caso concreto de proceso de negociación.

Sin embargo, Riley y McGinn (2002) subrayan que la ambigüedad no es suficiente para explicar los efectos de las diferencias de género en la negociación. Argumentan que es probable que las diferencias de género tengan un impacto significativo sobre el comportamiento o los resultados esperados. Pero esto es tan sólo una condición necesaria. Por tanto, las situaciones desencadenantes de la influencia de género constituyen un concepto clave a analizar.

Snyder y Ickes (1985) definen las situaciones desencadenantes de la influencia de género como aquellas en las que las diferencias individuales conllevan patrones distintos de respuesta. Por tanto, éstas conducen al cumplimiento de los estereotipos de comportamiento y performance. Estas situaciones "desencadenantes" o "precipitadoras" se han denominado "desencadenantes de la influencia de género" en el contexto de la negociación por Riley y McGuinn (2002) definiéndolos como "las características que hacen que las diferencias de género sean una cuestión patente y relevante, capaz de influir sobre el comportamiento y/o el resultado" (RILEY y MCGUINN, 2002:17). En particular, estos autores plantean dos tipos de desencadenantes de género para analizar el impacto sobre el resultado de la negociación: la estructura económica y el rol de representación.

La estructura económica de la negociación queda definida como el grado en el que el proceso representa una situación de suma fija o de suma variable de ganancias (RILEY y MCGUINN, 2002). Los autores proponen que cuando la negociación es de suma fija es de esperar que se establezca una interacción competitiva y, por tanto, surge o se hace necesaria la aparición del estereotipo de comportamiento masculino en la negociación. De acuerdo al Bem Sex-Role inventory (BSRI) (Bem, 1974) "masculino" está asociado a un comportamiento autosuficiente, competitivo e individualista. En contraste, un comportamiento "femenino" está asociado con características como son la sensibilidad hacia las necesidades de otros, la empatía, el entendimiento y la cooperación.

Por otro lado, en las situaciones caracterizadas por ser de suma variable, en las que se producen movimientos tanto cooperativos como competitivos (tensión crear-reclamar valor) (LAX y SEBENIUS, 1986), se espera la necesidad de comportamientos o atributos estereotipados tanto como masculinos como femeninos.

El segundo desencadenante de género propuesto es el rol de representación. Éste hace referencia a si las partes negociadoras negocian tratando de alcanzar sus propios intereses o si, por el contrario, se centran en la consecución de los objetivos comunes del colectivo al que representan. En este sentido, se argumenta que cuando un negociador negocia en nombre propio, el comportamiento es autosuficiente y no cooperativo. De este modo, se esperan negociaciones competitivas que favorecen la aparición de las diferencias de género, en este caso, que aparezca el patrón de carácter masculino.

En conclusión, desde una perspectiva situacional, el trabajo de Riley y McGuinn (2002) desarrolla una propuesta teórica centrada en el análisis de "cuándo" el género comienza a ser relevante en el proceso de negociación. Estos autores argumentan que los efectos de la influencia de género en la negociación se ven moderados por dos factores contextuales: la ambigüedad estructural y dos desencadenantes de género, a saber, la estructura económica de la negociación y el rol desempeñado por el agente en la negociación (representando o no a un colectivo). De acuerdo con su argumentación, se espera que si existen bajos niveles de ambigüedad estructural, el impacto de las diferencias de género sobre el comportamiento negociador y los resultados alcanzados 
no será significativo. Por el contrario, bajo condiciones de ambigüedad estructural es probable que el género tenga un efecto significativo tanto sobre el comportamiento como sobre los resultados del proceso negociador, pero sólo si el género es una cuestión importante para la interpretación de la negociación. Sin embargo, estos autores muestran que es posible que existan diferentes niveles de ambigüedad e incluso más factores desencadenantes de la influencia de género, diferentes a los analizados, que impliquen la existencia de efectos de género distintos sobre el proceso negociador.

\section{LA INFLUENCIA DE LAS DIFERENCIAS DE GÉNERO EN LOS PROCESOS DE NEGOCIACIÓN DE LOS ACUERDOS DE COOPERACIÓN}

Partiendo de la propuesta de Riley y McGuinn (2002), en el presente trabajo tratamos de analizar estos factores en un contexto específico: la negociación en los acuerdos de cooperación. De este modo, intentamos evitar las limitaciones de trabajos previos que, tal y como señala la literatura analizada, podrían haber generado la ambigüedad de los resultados obtenidos ante la no consideración del efecto del contexto.

Debido a, por un lado, el papel fundamental que juega el comportamiento negociador sobre los resultados y consecuencias de la implantación y desarrollo futuro de los acuerdos de cooperación y, por otro lado, el elevado ratio de fracaso que algunas modalidades de estos acuerdos experimentan, es importante analizar y comprender los efectos que algunos factores pueden tener sobre la elección del comportamiento negociador. Tal y como hemos señalado, uno de esos factores es el género.

Tal y como se indicó, los efectos de las diferencias de género sobre el comportamiento negociador serán significativas bajo condiciones de ambigüedad estructural. Así, la existencia de esta ambigüedad en el contexto de la negociación es esencial, aunque no suficiente, para estudiar esas diferencias (RILEY Y MCGUINN, 2002).

Los procesos de negociación de los acuerdos de cooperación son, por definición, ambiguos, y de ahí que los acuerdos alcanzados sean siempre incompletos. Resulta muy difícil prever todos los hechos futuros que podrían llegar a afectar o influir sobre la relación durante el desarrollo de las negociaciones (MENGUZZATO Y RENAU, 1995).

De este modo, ya que es casi imposible negociar cláusulas sobre todos los posibles factores que pudiesen aparecer en el futuro, el riesgo de que exista un comportamiento oportunista por alguna de las partes implicadas está siempre presente en los acuerdos de cooperación. Podemos decir, por tanto, que todos los acuerdos de cooperación poseen algunas características que los convierten en inciertos; de la misma manera, es factible pensar que la ambigüedad estructural está presente en todos los procesos de negociación.

Sin embargo, entendemos que, dependiendo del tipo de acuerdo y de sus características particulares, podemos encontrar diferentes niveles de ambigüedad estructural. En este sentido, por ejemplo, la joint venture se considera la modalidad de acuerdo de cooperación más compleja (a excepción de las redes de cooperación). Este proceso implica la creación de una nueva empresa con autonomía jurídica. De este modo, el número de asuntos que deben negociarse es muy elevado, conduciendo esto a una mayor complejidad del proceso ${ }^{5[3]}$. En este sentido, entendemos que como

\footnotetext{
${ }^{5[3]}$ En opinion de Carnevale, O'Connor y McCusker. (1993) y Stuhlmacher, Gillespie y Champagne, (1998) una negociación es más compleja cuando se deban negociar un mayor número de temas.
} 
consecuencia, el número de posibles hechos o problemas inesperados será más elevado, así como el nivel de incertidumbre.

Por el contrario, y contrastando con las negociaciones de las joint ventures, a nuestro modo de ver, el proceso de negociación de un acuerdo de franquicia es menos complicado, ya que está muy formalizado y cada aspecto del desarrollo de la relación es algo esperado y puede hacerse explícito. En un acuerdo de franquicia cada socio conoce lo que puede esperar del acuerdo (por ejemplo, beneficios económicos), hace explícitas sus exigencias y requerimientos (el franquiciado debe seguir requisitos estrictos relacionados, por ejemplo, con la decoración de la tienda o con las características de los vendedores) y ambos socios conocen cómo enfrentarse a las futuras situaciones de conflicto que pudieran tener lugar. En este caso, y ya que el grado de incertidumbre y ambigüedad es menor, el posible comportamiento oportunista de los socios estará bajo control (WILLIAMSON, 1985).

En este sentido, podemos formular una primera proposición:

Proposición 1: Cuanto mayor sea la complejidad del acuerdo de cooperación, mayor será la ambigüedad estructural del proceso de negociación.

Aparte de la ambigüedad estructural, el contexto en la negociación de un acuerdo de cooperación entendemos que viene caracterizado por los dos desencadenantes de género mencionados en la propuesta de Riley y McGinn (2002): la estructura económica de la negociación y el rol desempeñado en el proceso de negociación.

En el caso de los acuerdos de cooperación, la estructura económica de la negociación es de suma variable con periodos en los que la cooperación es necesaria (ganar-ganar) y otros periodos en los que se requiere actuar de modo competitivo. No obstante, ante las implicaciones mencionadas anteriormente sobre los resultados y la posibilidad de lograr una relación de confianza y de compromiso a largo plazo, cabe esperar un predominio de los movimientos cooperativos.

Igualmente, consideramos que se puede aplicar el segundo desencadenate de género propuesto por Riley y McGuinn (2002) en el contexto de los procesos de negociación de los acuerdos de cooperación. Así, por definición los negociadores en este tipo de contexto, participan representando a un colectivo (la empresa a la que representan) si bien somos conscientes que pudieran darse en ocasiones comportamientos oportunistas implicando, como consecuencia, costes de agencia (EISENHARDT, 1989).

En este sentido, ante la caracterización de los procesos de negociación de los acuerdos de cooperación como un contexto ambiguo, de suma variable y en el que las partes, en situaciones normales, supuestamente desempeñan el rol de representar a un colectivo, proponemos que:

Proposición 2: las diferencias en género ejercerán un impacto en los procesos de negociación de los acuerdos de cooperación, tanto sobre el comportamiento esperado como sobre los resultados de la negociación

Por tanto, ante el posible efecto de las diferencias en género sobre el comportamiento negociador $\mathrm{y}$, consecuentemente, sobre el resultado obtenido, y teniendo en cuenta las repercusiones sobre la correcta implantación de la estrategia, surge como algo esencial la necesidad de analizar la composición del equipo negociador. En este sentido, y como se mencionó, con la finalidad de alcanzar un buen resultado que facilite el compromiso al largo plazo, el equipo negociador debería estar formado por personas capaces de hacer 
frente correctamente a los distintos movimientos tanto de carácter cooperativo como competitivos que surgieran durante las negociaciones (adoptando en cada caso el tipo de comportamiento más adecuado).

Entendemos que el sexo biológico y los roles de género pueden ser cuestiones independientes, tal y como proponen Brewer, Mitchell y Weber, (2002). En este sentido, se distinguen tres estereotipos diferentes: masculino, femenino y andrógino (BEM, 1974). El rol "masculino" se asocia, en general, a un comportamiento autosuficiente y competitivo, y el rol "femenino" se relaciona con cuestiones como ser capaz de identificar las necesidades de los otros, empático, comprensivo/a y cooperativo/a. El trabajo de Portello y Long (1994) planteaba que una alta puntuación en las características propias del estereotipo "masculino" se traducía en comportamientos mucho más dominantes, competitivos y persuasivos; mientras que el perfil "andrógino" utilizaba un estilo de resolución de conflictos más integrador y colaborativo. De la misma manera, Jurma y Powell (1994) señalaban que los individuos que puntúan alto en el estereotipo femenino desarrollan mejores formas de comunicación que aquellos que poseen puntuaciones elevadas en el perfil que define el estereotipo "masculino".

Por tanto, siguiendo los planteamiento de Riley y McGuinn (2002) así como los últimos argumentos señalados, y entendiendo que el equipo negociador debe atender tanto a movimientos cooperativos como competitivos, proponemos que

Proposición 3: Con la finalidad de alcanzar un mejor resultado en las negociaciones de los acuerdos de cooperación (éxito), el equipo negociador debe estar compuesto por individuos con perfil andrógino

\section{PROPUESTA DE DISCUSIÓN}

El principal objetivo de este trabajo ha sido analizar el impacto de las diferencias de género sobre los procesos de negociación de los acuerdos de cooperación. Con esta finalidad nos hemos basado en la propuesta contextual de Riley y McGuinn (2002). En este sentido, entendemos que en el caso del contexto objeto de estudio -los acuerdos de cooperación-, las diferencias de género sí que ejercen una influencia significativa sobre el comportamiento negociador adoptado por las partes participantes, así como sobre el resultado que se obtiene en el proceso. Todo ello en razón de la caracterización de las negociaciones de los acuerdos de cooperación como contextos ambiguos, de suma variable y en el que se entiende que los negociadores participan en condición de representantes de un colectivo. Por tanto, y ante el impacto de las diferencias de género en nuestro contexto de análisis, proponemos que no cualquier composición de los equipos de negociación debiera considerarse como la más adecuada. Así, en aras a lograr un mejor resultado, que en el caso de los acuerdos de cooperación es aquel mutuamente satisfactorio al constituir la base de una relación de compromiso a largo plazo, la mejor composición de los equipos de negociación sería la de carácter andrógino. Esto es, los equipos de negociación deberían estar formados por personas que tuvieran un rol de comportamiento andrógino permitiendo, de este modo, la mejor gestión de todo tipo de movimiento (competitivo o cooperativo) que se produjera a lo largo del desarrollo de la negociación. Esto facilitaría la consecución de mejores resultados en términos de satisfacción con el acuerdo. 
Todas las relaciones propuestas entendemos que constituyen hipótesis a contrastar en futuras investigaciones, ya que nuestro trabajo es tan sólo un primer paso en el análisis de las relaciones entre género y comportamiento en los procesos de negociación. En cualquier caso, este trabajo ha tratado de sentar las bases de la discusión, planteando un modelo teórico que creemos puede contribuir a entender mejor el fenómeno analizado. Al mismo tiempo, permite reflexionar sobre la importancia de la composición de los equipos negociadores en función de la variable género, lo cual puede aportar nuevas ideas a los profesionales y directivos cuando deben elegir en quien confiar para llevar a cabo la negociación de un acuerdo, dadas las importantes repercusiones que dicha decisión tiene sobre el desarrollo y resultado del acuerdo.

\section{BIBLIOGRAFÍA}

ADLER, N.J; BRAHM, R.; GRAHAM, J.L. Strategy Implementation: A comparison of Face-to-Face Negotiations in the People's Republic of China and the United Status. Strategic Management Journal, 13: 449-466, 1992.

ALMER, E. What women need to know about starting up. The New York Times, 9, New York, 2000.

ARIÑO, A.; DE LA TORRE, J. Learning from Failure: Towards an Evolutionary Model of Collaborative Ventures, Organization Science, 9 (3): 306-325, 1998.

ARIÑO, A.; GARCÍA, C. Alianzas y bloques estratégicos: El comportamiento cooperativo como motor de su evolución, Revista Europea de Dirección y Economía de la Empresa, 5 (3): 133-144, 1996.

BABCOCK, L.; LOEWENSTEIN, G. Explaining bargaining impasse: The role of self-serving biases, Journal of Economic Perspectives, 11 (1): 109-126, 1997.

BEM, S.L. The measurement of psychological androgyny, Journal of Consulting and Clinical Psychology, 42 (2): 155-162, 1974.

BREWER, N.; MITCHELL, P.; WEBER, N. Gender role, organizational status, and conflict management styles, The International Journal of Conflict Management, 13: 78-94, 2002.

CARNEVALE, P.J.; PRUITT, D.G. Negotiation and mediation, Annual Review of Psychology, 43: 531-582, 1992.

CARNEVALE, P.J; O'CONNOR, K.M.; MCCUSKER, C. Time pressure in negotiation and mediation. In O. Suenson and A.J. Maule (eds), Time pressure and stress in human judgement and decision making, New York, pp: 117-126, 1993.

DYKMAN, B.M.; ABRAMSON, L.Y.; ALLOY, L.B.A.; HARTLAGE, S. Processing of ambiguous and unambiguous feedback by depressed and nondepressed college students: Schematic biases and their implications for depressive realism, Journal of Personality and Social Psychology, 56 (3): 431-445, 1989.

EISENHARDT, K. M. Agency Theory: an Assessment and Review. Academy of Management Review, 14 (1), pp. 57-74, 1989. 
FERNADEZ, I.; MEDINA, F.J.; DORADO, M.A. Características personales de los negociadores. In L. Munduate and F.J. Medina (eds), Gestión del conflicto, negociación y mediación. Pirámide, Madrid, pp: 201-228, 2005.

FERNANDES, E.; CABRAL-CARDOSO, C. The social stereotypes of the female manager and male manager: very alike but not enough, paper presented in the EURAM Congress, May, St. Andrews, (Scotland), 2004.

FISHER, R; URY, W.; PATON, B. Getting to Yes. Negotiating Agreement Without Giving In, Penguin Book, 1983.

FISKE, S.T.; TAYLOR, S.E. Social cognition, McGraw-Hill, New Cork, 1991.

GRAHAM, J.L. The Influence of Culture on Business Negotiations: An Exploratory Study", Journal of International Business Studies, 16 (1): 81-96, 1985.

GRAVES, L.M.; ELSASS, P.M. Sex and Sex dissimilarity effects in ongoing teams: Some surprising findings, Human Relations, 58 (2): 191-222, 2005.

HALPERN, J.J.; PARKS, J.M. Vive la difference: Differences between males and females in process and outcomes in a low conflict negotiation, The International Journal of Conflict Management, 7, 45-70, 1996.

HOCK, M.; KROHNE, H.W.; KAISER, J. Coping dispositions and the processing of ambiguous stimuli, Journal of Personality \& Social Psychology, 70 (5): 1052-1066, 1996.

JOHNSON, G.; SCHOLES, K.; WHITTINGTON, R. Exploring Corporate Strategy. Seventh edition, Prentice Hall, Harlow, 2005.

KLENKE, K. Gender influences in decision-making processes in top management teams, Management Decision, 41 (10): 1024-1034, 2003.

KORETZ, G. She's a woman, offer her less: A game sheds light on the pay gap, Business Week, May, 7: 34, 2001.

KRAY, L.J.; GALINSKY, A.D.; THOMPSON, L. Reversing the gender gap in negotiations: an exploration of stereotype regeneration, Organizational Behavior and Human Decision Processes, 87 (2): 386-409, 2002.

LAMBERT, A.J.; WEDELL; D.H. The self and social judgment: Effects of affective reaction and own position on judgements of unambiguous and ambiguous information about others, Journal of Personality \& Social Psychology, 61 (6): 884-897, 1991.

LAX, D.; SEBENIUS, J. The manager as a negotiator: Bargaining for cooperation and competitive gain, Free Press, New Cork, 1986.

LEWICKI, R.; LITTERER, J. Negotiation, Irwin, Homewood, IL, 1985.

LEWIN, K. A dynamic theory of personality, McGraw-Hill, New Cork, 1936.

MACLEOD, C.; COHEN, I.L. Anxiety and the interpretation of ambiguity: A text comprehension study, Journal of Abnormal Psychology: 102 (2): 238-247, 1993.

MENGUZZATO, M. La cooperación empresarial: un análisis de su proceso, IMPIVA, Valencia, Spain, 1992. 
MENGUZZATO, M.; RENAU, J.J. Estrategias de empresa y Teoría de los costes de transacción, Información Comercial Española, 746: 7-24, 1995.

MISCHEL, W. The interaction of person and situation, in D. Magnusson and N.S. Endler (eds), Personality at the crossroads: Current issues in interactional psychology, Erlbaum, Hillsdale, N.J., 1977.

MUNDUATE, L.; MEDINA, F.J. La naturaleza de la negociación. In L. Munduate, and F.J. Medina (eds), Gestión del Conflicto, Negociación y Mediación, Pirámide, Madrid:, pp: 119-136, 2005a.

. Planificación de la negociación. In L. Munduate L and F.J. Medina (eds), Gestión del Conflicto, Negociación y Mediación, Pirámide, Madrid:, pp: 137-156, 2005b.

NEU, J.; GRAHAM, J.; GILLY, M.C. The influence of gender on behaviour and outcomes in a retail buyer-seller negotiation simulation, Journal of Retailing, 64, 427-451, 1988.

PARGHI, I.G.; MURPHY, B.C. Gender and conflict resolution and negotiation: What the literature tells us, WAPPP (Women and Public Policy Program of Harvard's J.F. Kennedy School of Government. Student papers). www.ksg.harvard.edu/wappp/research/ working. 1999.

PORTELLO, J.; LONG, B. Gender role orientation, ethical and interpersonal conflicts and conflict handling styles of female managers. Sex Roles, 31: 683-701, 1994.

PRUITT, D.G. Negotiation Behavior, Academic Press, New York, 1981.

PRUITT, D.G.; CARNEVALE, P.J.D.; FORCEY, B.; VAN SLYCK, M. Gender effects in negotiation: Constituent surveillance and contentious behavior, Journal of Experimental Social Psychology, 22: 264-275, 1986.

PUTNAM, L.L. Reframing integrative and distributive bargaining: A process perspectiva. In B.H. Sheppard; M.H. Bazerman and R.J. Lewicki (eds), Research on Negotiation in Organizations, II, JAI Press, Inc., Beverly Hills, pp: 3-30, 1990.

RAO, A.; SCHMIDT, S.M. A behavioral perspective on negotiation international alliance, Journal of International Business Studies, 29 (4): 665-693, 1998.

RILEY, H.; MCGUINN, K.L. When does gender matter in negotiation?, working paper, Harvard University, 2002.

RING, P.S.; VAN DE VEN, A.H. Developmental Processes of cooperative Interorganizational Relationships, Academy of Management Review, 19 (1): 90-118, 1994.

ROSENTHAL, B.; RODRIGUES, M. Women-owned firms attract investors for business growth. National Foundation for Women Business Owners, Washington, D.C., 2000.

ROURE, J.B. Negociación. Resolución de problemas y Creación de valor, Folio, Barcelona, 1997.

RUBIN, J.Z; BROWN, B.R. The social psychology of bargaining and negotiation, Academic Press, New York, 1975.

SAORÍN, M.C. Choices in Joint Venture and Acquisition negotiation Behavior, Management Research, 2 (3): 219-234, 2004. 
SMELTZER, L.R.; WATSON, K.W. Gender differences in verbal communications during negotiation, Communication Research Reports, 3: 74-79, 1986.

SNYDER, M.; ICKES, W. Personality and social behaviour. In G. Lindzey and E. Aronson (eds), Handbook of social psychology, $3^{\text {rd }}$ ed., 2: 883-947, Random House, New York, 1985.

STEELE, C.M. A threat in the air: How stereotypes shape intellectual ability and performance, American Psychologist, 52: 613-629, 1997.

STEELE, C.M.; ARONSON, J. Stereotype threat and the intellectual test performance of African Americans. Journal of Personality and Social Psychology, 69: 797-811, 1995.

STUHLMACHER, A.F.; GILLESPIE, T.L.; CHAMPAGNE, M.V. The impact of time pressure in negotiation: A meta-analysis. International Journal of Conflict Management, 9 (2): 97-116, 1998.

THOMAS, P. Deals \& deal makers: At this camp women learn how to pitch to investors, The Wall Street Journal, Dulles, VA., 2000.

THOMPSON, L. Negotiation behavior and outcomes: Empirical evidence and theoretical issues, Psychological Bulletin, 108 (3): 515-532, 1990.

TJOSVOLD, D.; MORISHIMA, M.; BELSHEIM, J. Complaint handling on the shop floor: Cooperative relationships and open-minded strategies. International Journal of Conflict Management, 10 (1): 45-68, 1999.

URY, W., BRETT, J.M.; GOLDBERG, S.B. Getting Disputes Resolved: Designing Systems to Cut the Costs of Conflict, Jossey-Bass, San Francisco, 1988.

VALDÉS, A. Pautas para la creación y puesta en marcha de las empresas conjuntas, Alta Dirección, 199: 195-203, 1998.

VERHEUL, I.; RISSEEUW, P.; BARTELSE, G. Gender Differences in Strategy and Human Resource Management: The Case of Dutch Real Estate Brokerage, Sage, Thousand Oaks, 2002.

WALTER, A.E.; STUHLMACHER, A.F.; MEYER, L.L. Gender and negotiator competitiveness: A meta-analysis. Organizational Behavior and Human Decision Processes, 76 (1): 1-29, 1998.

WEISS, S.E. Analysis of Complex Negotiations in International Business: The RBC Perspective, Organization Science, 4 (2): 269-300, 1993.

WEISS, S.E. Explaining outcomes of negotiation: Toward a grounded model for negotiations between organizations. In R.J. Lewicki; R.J. Bies and B.H. Sheppard (eds), Research on Negotiation in Organizations, JAI Press, pp: 247-333, 1997.

WILLIAMSON, O.E. The Economic Institutions of Capitalism: Firms, Markets, Relational Contracting, Free Press, New York, 1985. 


\title{
GENDER DIFFERENCES IN THE NEGOTIATION OF COOPERATION AGREEMENTS AND THEIR INFLUENCE ON THE PROCESS RESULTS
}

\begin{abstract}
The relationships between gender and behavior, and the expected behavior under this stereotypy, is a key question that this paper intends to answer. More specifically, we analyze the influence of gender differences and the behavior that arises from these differences on the results of the negotiation processes. With the objective to control the influence of the context where the negotiation process is produced, we focused in the negotiation of cooperation agreements. This kind of agreement is characterized by an elevated degree of structural ambiguity, the negotiations are variable and the agents act in name of the collective they represent. In situations like this the gender differences become apparent and conduct to a reflection about the ideal composition of the negotiation group. The negotiation group should be composed by an androgen profile, capable of adopting both, cooperative and competitive behaviors according to the situation.
\end{abstract}

Key words : Gender, negotiation behavior, negotiation results, negotiation team, strategy alliances. 